capacity. Span has recently announced that they can market the Spirolipin dual test for $<1$ USD per cassette, which would make these tests affordable in many settings. Future developments in rapid testing include the addition of antigen(s) to also test for HIV infection, offering even more diagnostic options to control these diseases, especially among pregnant women.

\section{S4.5 MATERNAL SYPHILIS TESTING AND TREATMENT TO IMPROVE MATERNAL AND CHILD HEALTH SERVICES: PROGRESS AND CHALLENGES}

doi:10.1136/sextrans-2011-050102.18

\section{Kamb. CDC, Atlanta, Georgia, USA}

Background In 2007, WHO released the document Global Elimination of Congenital Syphilis, Rationale and Strategy for Action, developed in collaboration with a team of expert consultants. The strategy aims to prevent mother to child transmission of syphilis through the strengthening of antenatal care programs to ensure:

Early antenatal care for all women, with universal syphilis screening and prompt treatment of those infected

Treatment of all sexual partners of infected women, promotion of condom use during pregnancy, and counselling of all women on how to prevent infection

All neonates born to RPR-positive mothers are given a single dose of penicillin as prophylactic treatment.

This presentation documents progress in the global elimination initiative thus far, notes challenges towards substantial reduction in congenital syphilis by 2015 , and proposes areas for focus in the next 2 years.

Methods/results Most progress has been made in areas of advocacy and recognition of the public health problem, the emergence of rapid diagnostic tests and (in some areas of the world) integrated intervention initiatives such as those linking prevention of mother to child transmission of HIV and congenital syphilis. Progress has also been made in integration of a handful of critical indicators into existing data collection systems, allowing better monitoring of national and global program progress. Publication of program evaluations has been mixed, with increased reporting on evaluations of implementing testing and treatment strategies, but still limited research on validation of proposed impact targets (eg, use of stillbirth as an indicator of impact). Additionally, specific funding remains limited, although an Investment Case for donors is near completion and some new opportunities for funding integrated services have arisen.

Conclusions Some emerging global issues such recent initiatives and trends in global public health financing may influence future progress. National guidelines on testing strategies are needed to avoid less than optimal or more costly than needed testing strategies. More focused efforts on earlier and higher quality antenatal care will likely support congenital syphilis elimination along with promoting better overall antenatal services.

\section{Symposium 5: Unsolved challenges in women's sexual and genital health

S5.1 THE OTHER VAGINITIDES: UPDATE ON DESQUAMMATIVE
INFLAMMATORY VAGINITIS AND REFRACTORY CANDIDA
VAGINITIS

doi:10.1136/sextrans-2011-050102.19

\section{J D Sobel. Wayne State University School of Medicine, Detroit, USA}

Desquammative Inflammatory Vaginitis (DIV) is now recognised as a not uncommon caused of purulent vaginitis with a profuse discharge accompanying dyspareunia and vaginal burning. Less well recognised are the vestibular and vulvar manifestations of this chronic idiopathic and likely immune mediated inflammatory process which occurs exclusively in Caucasian women usually in the peri-menopausal period. The syndrome must be differentiated from trichomoniasis since both share several clinical manifestations including markedly elevated vaginal $\mathrm{pH}$ and inflammatory cell infiltrate, but several features to be discussed allow correct diagnosis.

DIV is likely precipitated by oestrogen deficiency explaining it's unique epidemiology. Treatment consisting of high potency steroids and other local anti-inflammatory agents results in rapid improvement but cure frequently requires prolonged long term therapy.

A new clinical entity of recurrent vaginitis due either to drug resistant Candida albicans and other Candida species including Candida glabrata will be discussed. These frustrating cases constitute a major therapeutic challenge because of intrinsic or acquired fluconazole resistance and frequent cross resistance to the entire Azole drug class. Therapeutic options are few, moreover, in vitro breakpoints defining Azole drug resistance have not been established. Prolonged exposure to fluconazole appears responsible. Cases will be presented describing several forms of refractory drug resistant vaginal candidiasis.

\section{S5.2 ACTION HEROES OR CASUAL OBSERVERS: VAGINAL MICROFLORA AND STI/HIV RISK}

doi:10.1136/sextrans-2011-050102.20

S Hillier. University of Pittsburgh School of Medicine, Pittsburgh, USA

Some components of the microbial flora of the vagina, most notably lactobacilli, have been linked with decreased rates of bacterial and viral STIs, including HIV, mucopurulent cervicitis and pelvic inflammatory disease. However, the same sexual activity which adversely impacts vaginal colonisation by lactobacilli increases risk of STIs. Therefore, the apparent protective benefits of lactobacilli could reflect co-epidemiology rather than an independent biological benefit of these microorganisms. This presentation will present a summary of the epidemiological data linking vaginal microflora to HIV/STI risk and evidence suggesting that lactobacilli play a causal role in reducing risk of STI/HIV in women.

\section{S5.3 NEW DATA ON PELVIC INFLAMMATORY DISEASE: IMPLICATIONS FOR SCREENING AND MANAGEMENT}

doi:10.1136/sextrans-2011-050102.21

J Ross. British Association for Sexual Health and HIV-BASHH, London, UK

Pelvic inflammatory disease is a common cause of morbidity in young sexually active women, but many of our preventive efforts and management strategies are based on empirical approaches. Central to the implementation of chlamydia screening programmes is an understanding of the risk of PID following chlamydial infection. The recent POPI study helps to inform this risk and also provides information about the role of other potential pathogens. The importance of Mycoplasma genitalium as a cause of PID needs to be clarified, but its implications for testing and treatment are now becoming clearer. Women with PID are often poorly adherent to antimicrobial treatment and recent trials suggest that newer treatment regimens may be easier to take and have the potential to improve outcomes. 